\title{
ANÁLISE COMPARATIVA DA EFICIÊNCIA ECONÔMICA E COMPETITIVIDADE DOS CULTIVOS DE CAMARÃO MARINHO NO SISTEMA SEMI-INTENSIVO (TRADICIONAL) E SUPERINTENSIVO (COM REUSO DE ÁGUA E USO DE BIOFLOCOS - BFT) UTILIZADOS NO BRASIL
}

\author{
João Felipe Nogueira Matias \\ jfn.matias@gmail.com \\ Universidade Federal Fluminense - \\ UFF, Niterói, Rio de Janeiro, Brasil
}

Halana Rodrigues Freire Floy

halanarodrigues@gmail.com Fundação Cearense de Apoio ao Desenvolvimento Científico e Tecnológico - FUNCAP, Fortaleza, Ceará, Brasil

\section{Karla Maria Catter}

kmcatter@yahoo.com.br Fundação Cearense de Apoio ao Desenvolvimento Científico e Tecnológico - FUNCAP, Fortaleza, Ceará, Brasil

Rochelle Cruz de Araújo Bezerra Vidigal

rochelle.cruz@cedepesca.net Fundação Cearense de Apoio ao Desenvolvimento Científico e Tecnológico - FUNCAP, Fortaleza, Ceará, Brasil

\section{Rossi Lelis Muniz de Souza}

rossilelis@gmail.com

Fundação Cearense de Apoio ao Desenvolvimento Científico e Tecnológico - FUNCAP, Fortaleza, Ceará, Brasil

\section{Viviana Lisboa}

viviana.lisboa.lisboa@gmail.com Fundação Cearense de Apoio ao Desenvolvimento Científico e Tecnológico - FUNCAP, Fortaleza, Ceará, Brasil

\section{Mariana Lopez Matias} marilopez.matias@gmail.com Universidade de Fortaleza, Fortaleza, Ceará, Brasil

\section{RESUMO}

A carcinicultura marinha (cultivo de camarões marinhos) tem uma enorme importância socioeconômica no Nordeste brasileiro, pois é nesta região que se concentram $98 \%$ da produção de camarão cultivado no Brasil. Porém, esta atividade vem sofrendo com baixa produtividade e perda de competitividade dos atuais sistemas de cultivo. Este artigo teve como objetivo realizar uma análise comparativa entre a eficiência econômica e a competitividade dos cultivos de camarão marinho no sistema semi-intensivo (tradicional) e no sistema superintensivo (reuso de água e uso de bioflocos - BFT) utilizados no Brasil. Foi feita uma comparação entre os indicadores de eficiência econômica e competitividade em cada um dos sistemas de cultivo de camarões marinhos estudados. Os dados necessários para realizar esta análise comparativa foram coletados por meio de levantamentos bibliográficos junto às fontes secundárias. Os resultados obtidos demonstram que o cultivo de camarões marinhos no sistema com reuso de água e BFT é, no que se refere aos parâmetros econômicos, mais eficiente e competitivo do que o cultivo no sistema semi-intensivo (tradicional). As conclusões deste trabalho nos permitem aprofundar as discussões a respeito da modernização da carcinicultura brasileira por meio da intensificação destes cultivos. É necessário que os estudos tragam à tona a adoção de inovações tecnológicas que proporcionem uma melhoria na eficiência, na competitividade da aquicultura brasileira e que sejam incentivados. A originalidade deste trabalho reside neste fundamento.

Palavras-chave: carcinicultura superintensiva, bioflocos, eficiência, competitividade 


\section{INTRODUÇÃO}

O mundo vive uma nova ordem mundial que surgiu a partir da pandemia originada pelo novo coronavírus. Além de toda a crise humanitária e de saúde, os impactos econômicos ainda serão sentidos de forma bastante contundente nos próximos anos. Os países terão que concentrar esforços que proporcionem a retomada da atividade econômica, a geração de trabalho e renda, produção de alimentos e o acesso da população a estes alimentos. Desta forma, a aquicultura (cultivo de organismos aquáticos) pode vir a contribuir enormemente com estes fatores.

A Food and Agriculture Organization of the United $\mathrm{Na}$ tions (FAO, 2020) cita que a produção mundial de pescado para consumo humano em 2018 foi de 156 milhões de toneladas ( $\mathrm{t}$ ), sendo que a aquicultura foi responsável por $52 \%$ deste total, isto é, 82 milhões $t$. Por sua vez, os crustáceos, como o camarão, foram responsáveis por 9,4 milhões $t$, representando quase US\$ 70 bilhões.

No Brasil, a aquicultura produziu 579 mil t em 2018, das quais 43 mil $t$ foram de cultivo de camarões marinhos (carcinicultura) (IBGE, 2019). A crescente demanda pelo produto no mercado externo durante o começo dos anos 2000 e o advento de novas tecnologias para cultivar o camarão marinho em água doce impulsionou a entrada de novos adeptos à atividade (Souza Junior, 2003). Assim, a importância da cadeia agroindustrial do camarão cultivado aumentou em virtude do número de carcinicultores dedicados à atividade, pela extensão das áreas ocupadas com a carcinicultura, pelo valor da produção e pela capacidade de gerar empregos, contribuindo para o desenvolvimento local (Souza Junior, 2003).

Os sistemas utilizados pelos produtores podem ser divididos, em função da produtividade, em extensivo, semi-intensivo ou intensivo; por número de espécies envolvidas (monocultura ou policultura); e conforme o compartilhamento em consórcio com outras espécies que não aquelas exclusivamente aquáticas (Oliveira, 2009). O cultivo extensivo refere-se à exploração feita em açudes, lagoas, represas e outros mananciais, nas quais não há interferência contra predadores, qualidade da água e alimento. No semi-intensivo há interferência em relação ao alimento e à fertilização da água com suplementos. E para o intensivo, utilizam-se rações balanceadas em virtude da alta densidade de indivíduos. Assim, quanto menor as interferências nas condições do cultivo, menor será a produtividade (Oliveira, 2009).

Na primeira metade dos anos 1990, os laboratórios brasileiros dominaram a reprodução e a larvicultura do $L$. vannamei, iniciando a distribuição comercial de póslarvas. 0 Litopenaeus vannamei apresentou viabilidade comercial, com índices de produtividade e rentabilidade superiores aos das espécies nativas, tornando-se a única espécie cultivada atualmente em sistemas de produção semi-intensivo, maneira mais adequada às condições dos estuários brasileiros (Souza Junior, 2003).

Diante da importância de investir em tecnologias limpas que permitam a redução de inputs de nutrientes e taxas de renovação de água para favorecer equilíbrio aos ambientes adjacentes, surgiram tecnologias capazes de produzir de forma diferente (Nascimento et al., 1998). Os cultivos sem renovação de água ZEAH (Zero Exchange, Aerobic, Heterotrophic Culture Systems) ou cultivo em meio aos Bioflocos (BFT), por exemplo, vem ao encontro dos novos conceitos de uma aquicultura responsável e ambientalmente correta, pois são realizados praticamente sem renovação de água e com aproveitamento dos microrganismos como alimento natural, o que reduz o uso de ração (Sampaio et. al., 2010). Já o sistema BFT, além de melhorar os índices de produtividade, comparado com os sistemas tradicionais de cultivo, apresenta maior biossegurança, pois diminui trocas de água, evitando doenças. Este tipo de sistema utiliza pouca água, representando uma diminuição na emissão de efluentes, podendo produzir $1 \mathrm{~kg}$ de camarão com a utilização de menos de 160 litros de água (Otoshi et al., 2006), enquanto nos sistemas convencionais é utilizado até 64.000 litros (Hopkins et al., 1995).

Assim, diante da importância que a carcinicultura ganhou na economia do Nordeste, o presente estudo tem o objetivo de comparar a eficiência econômica e competitividade entre os cultivos de camarão marinho realizados no sistema tradicional (semi-intensivo) e no sistema de recirculação de água, com o uso de BFT.

\section{ANÁLISE DA EFICIÊNCIA ECONÔMICA E COMPETITIVIDADE}

Em toda análise de viabilidade econômica é salutar estudar o ponto a partir do qual a empresa se torna lucrativa. Esse ponto recebe o nome de ponto de equilíbrio ou break even point (Coelho, 2005). Segundo Gitman (1997), a análise do ponto de equilíbrio é usada pela empresa para determinar o nível de operações necessárias para cobrir todos os custos operacionais e para avaliar a lucratividade associada a vários níveis de venda. Seguindo nesta linha, Hoji (2001) afirma que, no ponto de equilíbrio, a empresa está produzindo e vendendo a quantidade de produtos suficiente para cobrir os custos, além de despesas totais.

Para a análise econômica da atividade pela estimativa do custo de produção, é necessário utilizar indicadores de eficiência econômica, tais como a margem bruta, margem líquida, resultado (lucro ou prejuízo) e subsídios interessantes para a tomada de decisões na empresa agrícola. A margem 
bruta é utilizada considerando que o produtor possua os recursos disponíveis (terra, trabalho e capital) e a necessidade de tomar decisões de como utilizar, de forma eficaz, esses fatores de produção. A margem líquida permite concluir se a atividade é estável, com possibilidade de expansão e de se manter por longo prazo, quando essa for positiva. Se a margem líquida for igual a zero, a propriedade estará no ponto de equilíbrio e em condições de refazer, em longo prazo, seu capital fixo. Mas, se ela for negativa, significa que o produtor poderá continuar produzindo por um determinado período, embora com um problema crescente de descapitalização.

Adaptando o conceito de ponto de equilíbrio para os projetos desenvolvidos por uma empresa, é possível estimar a partir de que momento o projeto começa a ser lucrativo. Algumas técnicas são utilizadas para calcular o retorno estimado de um investimento, haja vista que as despesas realizadas hoje somente trarão resultados positivos após algum tempo depois de iniciada a realização. As técnicas mais comuns para a análise são as do Período de Payback (PP), Valor Presente Líquido (VPL) e Taxa Interna de Retorno (TIR), citadas em muitas obras de finanças, entre as quais as de Gitman (1997) e Hoji (2001).

O PP, por exemplo, é o método que analisa o tempo de recuperação do dinheiro investido, ou seja, quanto tempo levará para que a empresa recupere o dinheiro investido. $\mathrm{Na}$ questão do PP, quanto maior for o tempo de payback, maior será a incerteza e os riscos ao retorno do investimento. Desse modo, quanto menos tempo, maior a probabilidade de acontecer o retorno do investimento (Santos; Vasan, 2014).

No que diz respeito ao VPL, este é utilizado para calcular o valor presente de uma série de pagamentos futuros descontado a uma taxa de custo de capital estipulada. Este método considera o fato de que o dinheiro que vamos receber no futuro não tem o mesmo valor do dinheiro no tempo presente, haja vista a incerteza do amanhã. O VPL determina, portanto, a quantidade monetária a qual o projeto irá aumentar o valor da sua empresa. Deve-se estimar, assim, o valor do custo do projeto e a sua renda futura (Santos; Vasan, 2014).

Outra técnica bastante utilizada é a TIR, que tem o objetivo de determinar a partir de que percentual de retorno haverá lucro para o projeto. A taxa indica quando o valor atual do seu projeto será igual a zero. Ou seja, o projeto tornar-se-á interessante quando a TIR for maior que o custo de capital do seu projeto (Santos; Vasan, 2014). Essa taxa representa a remuneração do capital investido e permite identificar o risco de investimento de um projeto, haja vista a facilidade de se comparar a TIR de um determinado projeto com a taxa de uma aplicação financeira de baixo risco, por exemplo.

Percebe-se que as técnicas apresentadas são úteis para a análise da rentabilidade do investimento, uma vez que as estimativas preveem o valor necessário para a implementação do projeto (considerando-se os custos totais de operação) e o valor que irá retornar ao investidor, após a devida execução. Por definição, a rentabilidade é a relação entre o valor do retorno e valor investido, proporção que permite ao produtor comparar, dentre os projetos disponíveis, qual deles traz o maior benefício.

\section{O CULTIVO DE CAMARÕES MARINHOS NO BRASIL}

No censo mais recente da carcinicultura brasileira, a Associação Brasileira de Criadores de Camarão (ABCC, 2015) relata que esta cadeia produtiva contava com 2.000 fazendas de engorda em 2014, ocupando uma área de 23.000 hectares (ha), totalizando uma produção de 85.000 t de camarões; 32 unidades de maturação e larvicultura, com uma produção de 20 bilhões de pós-larvas; nove fábricas de ração, com uma produção de 126.000 t/ano e; 32 unidades de beneficiamento, com uma produção de 40.000 t/ano. A Tabela 1 apresenta a receita oriunda das atividades envolvidas na cadeia produtiva da carcinicultura em 2014.

Tabela 1. Receita da Cadeia Produtiva da Carcinicultura Marinha em 2014

\begin{tabular}{|c|c|}
\hline Atividade & Receita (R\$) \\
\hline Fazendas de engorda & $1.350 .000 .000,00$ \\
\hline Maturação e larvicultura & $170.000 .000,00$ \\
\hline Fábricas de ração & $378.000 .000,00$ \\
\hline Beneficiamento & $130.000 .000,00$ \\
\hline Total & $2.028 .000 .000,00$ \\
\hline
\end{tabular}

Fonte: ABCC (2015)

Já de acordo com o último levantamento sobre a infraestrutura produtiva e os aspectos tecnológicos, econômicos, sociais e ambientais da carcinicultura marinha no Brasil (ABCC, 2013), é na área rural costeira da região Nordeste que está situada a maioria expressiva das fazendas de camarão marinho do Brasil (Tabela 2), especialmente nos estados do Ceará com 31.982 t, Rio Grande do Norte com 17.825 t, Bahia com 7.050 t, Pernambuco com 4.309 t, Piauí com 3.079 t, Sergipe com 2.973 t e Paraíba com 1.530 t. Esses estados respondem por $98,8 \%$ da produção brasileira.

Tabela 2. Carcinicultura no Brasil por região

\begin{tabular}{|c|c|c|c|}
\hline Região & No fazendas & $\begin{array}{c}\text { Área cultivável } \\
\text { (ha) }\end{array}$ & Produção (t) \\
\hline Nordeste & 1.429 & 20.866 & 69.171 \\
\hline Sul & 112 & 1.346 & 344 \\
\hline Norte & 3 & 33 & 56 \\
\hline Sudeste & 1 & 103 & - \\
\hline
\end{tabular}

Fonte: ABCC (2013) 
Ainda segundo os dados da ABCC (2013), embora esta atividade tenha crescido em relação ao número de produtores e à área cultivada, decresceu em termos de produção e produtividade no período 2004-2011, conforme dados apresentados na Tabela 3.

Tabela 3. Dados comparativos da carcinicultura brasileira (2004, 2011)

\begin{tabular}{|c|c|c|c|}
\hline Variável & $\mathbf{2 0 0 4}$ & $\mathbf{2 0 1 1}$ & Variação \\
\hline No de produtores & 997 & 1.545 & $55 \%$ \\
\hline Área (ha) & 16.598 & 22.347 & $35 \%$ \\
\hline Produção (t) & 75.904 & 69.571 & $-8 \%$ \\
\hline Produtividade (t/ha) & 4,51 & 3,51 & $-22 \%$ \\
\hline \multicolumn{4}{|c}{ Fonte: ABCC, 2013 }
\end{tabular}

\section{O cultivo de camarões marinhos no sistema semi- intensivo (tradicional)}

De acordo com Nunes et al. (2011), no período entre 1998 e 2003, a carcinicultura brasileira teve seu momento de auge com a instalação de novos empreendimentos, modernização da infraestrutura existente, incorporação de tecnologias e controles ao cultivo, ao passo que o período entre 2004 e 2011 pode ser dividido em duas etapas distintas: a) o ajuste a uma nova realidade produtiva e econômica; e b) a recuperação econômica do setor, com a retomada da produção e consolidação da indústria.

No primeiro período ocorreram adversidades que comprometeram as atividades, dentre elas, enfermidades do animal, queda dos preços do camarão no mercado internacional (em virtude do aumento da produção asiática), desvalorização progressiva do dólar, ação antidumping dos Estados Unidos, aumento dos custos operacionais no modelo intensivo e diminuição das produtividades.

A partir de 2005, na busca por soluções a esses problemas, os projetos que se mantiveram ativos reduziram seus custos em detrimento das altas produtividades, antes almejadas. Na carcinicultura, os custos operacionais (ração, pós-larva, energia e mão-de-obra) estão atrelados ao nível de intensificação. Com isto, os produtores baixaram drasticamente as densidades de estocagem, passando a operar com densidade compreendida entre 10 e 15 camarões $/ \mathrm{m}^{2}$. Além disso, os tempos de engorda foram reduzidos e houve uma busca dos produtores pelo mercado interno. Outro fato que contribuiu para a recuperação foi o desconto de energia elétrica concedido ao setor, por meio da Resolução Normativa no 207, editada pela Agência Nacional de Energia Elétrica (ANEEL) em 9 de janeiro de 2007, proporcionando a alteração do cenário a partir de 2008.

A partir deste momento, a atividade retomou seus índices de rentabilidade, beneficiada pelo novo cenário econômico do país, que incorporou uma massa significativa de pessoas com poder de compra. Enquanto a produção se estabilizou ao redor de 65 mil t, o consumo per capta de camarões no Brasil aumentou significativamente. Ainda segundo Nunes et al. (2011), entre os fatores técnicos que mais contribuíram na recuperação econômica da atividade é o retorno à predominância do sistema de cultivo semiintensivo, no qual a redução das densidades de estocagem e o tratamento do solo, por meio de biorremediadores, promoveram uma significativa diminuição das condições de estresse, além da redução na frequência de doenças. A Tabela 4 registra as densidades de estocagem das fazendas de cultivo de camarão marinho em 2011 (ABCC, 2013).

Observa-se que quase $90 \%$ dos produtores de camarão utilizaram uma densidade de estocagem de até 30 camarões $/ \mathrm{m}^{2}$; menos de $9 \%$ utilizaram uma densidade entre 30 e 50 camarões $/ \mathrm{m}^{2}$; e somente $1,7 \%$ utilizam densidades de estocagem maiores do que 50 camarões $/ \mathrm{m}^{2}$.

\section{O cultivo de camarões marinhos no sistema super intensivo (com reuso de água e uso de bioflocos - BFT)}

Devido à problemática do uso da água e a poluição dos ambientes aquáticos costeiros, muita atenção tem sido dada a iniciativas para que otimizem o uso deste recurso e que as atividades dependentes da água sejam cada vez mais sustentáveis. E para isso, tem-se utilizado a intensificação dos cultivos. De acordo com Teixeira e Guerrelhas (2011), o sistema de cultivo intensivo possui algumas características: a) demanda acompanhamento contínuo e intenso; b) permite aumentar a produtividade em três vezes ou mais e não aumenta o custo de produção na mesma intensidade; c) densi-

Tabela 4. Categoria de produtor $X$ densidades de estocagem

\begin{tabular}{|c|c|c|c|c|c|}
\hline Categorias & $\mathbf{N}^{\mathbf{0}}$ de Produtores & $<$ que $\mathbf{1 0} \mathbf{~ c a m} / \mathbf{m}^{\mathbf{2}}$ & Entre $\mathbf{1 0}$ e $\mathbf{3 0} \mathbf{~ c a m} / \mathbf{m}^{\mathbf{2}}$ & Entre $\mathbf{3 0}$ e $\mathbf{5 0} \mathbf{~ c a m} / \mathbf{m}^{\mathbf{2}}$ & $\mathbf{>} \mathbf{q u e} \mathbf{5 0} \mathbf{~ c a m} / \mathbf{m}^{\mathbf{2}}$ \\
\hline Micro & 717 & 342 & 315 & 35 & 11 \\
\hline Pequeno & 184 & 78 & 72 & 29 & 5 \\
\hline Médio & 245 & 80 & 130 & 28 & 3 \\
\hline Grande & 76 & 14 & 47 & 12 & 2 \\
\hline Total & 1222 & $513(42,7 \%)$ & $564(46,9 \%)$ & $104(8,7 \%)$ & $21(1,7 \%)$ \\
\hline
\end{tabular}


dades mais altas podem suportar altas taxas de crescimento, desde que a ração tenha composição para tal, os níveis de oxigênio sejam mantidos em níveis ideais, a condição ambiental do viveiro seja estável e controlável.

Dentro desta linha, sistemas do tipo fechado (recirculação de água) têm sido amplamente testados e difundidos, principalmente pela maior biossegurança, maior sustentabilidade ambiental, além das vantagens adquiridas através da intensificação da produção quando comparadas a sistemas convencionais extensivos e semi-intensivos, no qual ocorrem trocas de água.

Neste cenário, um sistema de cultivo que vem sendo amplamente estudado e difundido é a "Tecnologia de Bioflocos" (Biofloc Technology - BFT), que possui como principal característica a não renovação de água. No sistema BFT, compostos tóxicos oriundos da excreção dos camarões e dos restos de ração presentes no meio são convertidos em biomassa bacteriana por meio da ação de bactérias heterotróficas e nitrificantes.

Estes agregados microbianos, denominados bioflocos, possuem um importante papel, que é a transformação dos compostos tóxicos em alimento para os camarões. O cultivo no sistema BFT favorece uma maior intensificação do sistema de colheita em decorrência da manutenção da qualidade da água e do suplemento alimentar natural existente.

Vários estudos têm demonstrado os inúmeros benefícios do consumo dos bioflocos pelos animais cultivados, tais como: redução nas taxas de conversão alimentar, aumento das taxas de crescimento, fortalecimento do sistema imune e diminuição considerável dos custos com ração. Até $50 \%$ do alimento consumido pelo camarão $L$. vannamei neste sistema de cultivo é composto por bioflocos.

\section{MÉTODO}

Foi realizada uma pesquisa descritiva bibliográfica documental. Este tipo de pesquisa, segundo Bastos (2012), descreve a situação no momento que ocorre a investigação e estabelece relação entre as variáveis.

Além disso, foi utilizada uma abordagem quali-quantitativa. De acordo com Richardson (1989) apud in Bastos (2012, p. 41), o aspecto qualitativo de uma investigação pode estar presente até mesmo nos dados coletados por estudos quantitativos, sem perderem seu caráter qualitativo ao serem transformados em dados quantificáveis, com o objetivo de assegurar a exatidão dos resultados.

Inicialmente foi realizado um levantamento bibliográfico que possibilitou a apresentação de conceitos e metodolo- gias que permitem mensurar a eficiência econômica, além da competitividade em atividades produtivas.

Após essa fase, foi realizado outro levantamento bibliográfico, desta vez para apresentar as características gerais do cultivo de camarões marinhos utilizados no Brasil, assim como para descrever o sistema tradicional (semi-intensivo) de cultivo de camarões e o sistema de cultivo com recirculação de água, com o uso de bioflocos.

Ambos os levantamentos foram feitos por meio de fontes secundárias, estudos prévios e informações estatísticas em instituições de pesquisa, empresas, associações, cooperativas, órgãos governamentais e outras fontes detentoras de dados, que permitissem realizar uma comparação da eficiência econômica e competitiva dos sistemas de cultivo de camarões marinhos utilizados neste trabalho.

Os dados coletados foram de parâmetros de eficiência econômica, como custos operacionais, preço médio, margem de lucro, ponto de equilíbrio, rentabilidade simples, payback, TIR e VPL, referentes a cada sistema de cultivo estudado. De posse desses dados, foi possível realizar a análise comparativa entre os dois sistemas de cultivo, objetivo geral deste estudo.

\section{RESULTADOS}

Para fins de comparação entre os sistemas de cultivo de camarão marinho trabalhados neste artigo, foram identificadas as premissas mais relevantes referentes à cada sistema de cultivo.

Em seguida, apresenta-se a análise de cada sistema discutido separadamente, sendo o tradicional (semi-intensivo) e o superintensivo (com recirculação de água e uso de bioflocos), para finalmente realizar uma análise comparativa entre os resultados encontrados.

\section{Sistema semi-intensivo (tradicional)}

Premissas:

- Área de cultivo (engorda): 50 hectares

- Valor do investimento: $\mathrm{R} \$ \mathbf{1}$ 1.350.000,00

- Densidade de cultivo na engorda: 25 camarões por $\mathrm{m}^{2}$.

- Conversão alimentar: 1,8 kg de ração: 1,00 kg de camarão 


\section{- Produtividade: $6.843 \mathrm{~kg} /$ hectare/ano}

Os resultados encontrados neste trabalho para o sistema de cultivo tradicional (semi-intensivo) nos mostram que seriam necessários 50 hectares de engorda de camarão, com um investimento de $\mathrm{R} \$ 1.350 .000,00$ para se obter: ponto de equilíbrio de $8,15 \%$, rentabilidade simples de $238,19 \%$, payback de 0,42 ano, TIR de 153,24\% e VPL de R\$13.847.343,60 a $12 \%$ ao ano. Todos estão representados na Tabela 5:

Tabela 5. Resultados dos parâmetros econômicos para o cultivo de camarão marinho numa área de 50 hectares, no sistema tradicional (semi-intensivo)

\begin{tabular}{|c|c|}
\hline Parâmetros & Resultados \\
\hline Custo de Produção (R\$/ kg) & 17,17 \\
\hline Preço Médio (R\$) & 30,00 \\
\hline Margem de Lucro (R\$) & 12,83 \\
\hline Ponto de equilíbrio (\%) & $8,15 \%$ \\
\hline Rentabilidade Simples (\%) & 238,19 \\
\hline Payback (ano) & 0,42 \\
\hline Taxa Interna de Retorno (\%) & 153,24 \\
\hline Valor Presente Líquido a 12\% a.a. (R\$) & $13.847 .343,60$ \\
\hline
\end{tabular}

Fonte: Dados da Pesquisa

\section{Sistema superintensivo (reuso de água e uso de bioflocos - BFT)}

Para fins de comparação entre os sistemas de cultivo de camarão marinho trabalhados neste artigo, foram identificadas as premissas mais relevantes referentes à cada sistema de cultivo.

\section{Premissas:}

- Área de cultivo (engorda): 01 hectare.

- $\quad$ Valor do investimento: $\mathrm{R} \$ 700.000,00$

- Densidade de cultivo na engorda: 180 camarões/m².

- Conversão alimentar: 1,3 kg de ração: 1,0 kg de camarão

- Produtividade: $96.821 \mathrm{~kg} /$ hectare/ano

Os resultados encontrados neste trabalho para o sistema de cultivo com recirculação de água e com o uso de BFT nos mostra que seria necessário um hectare de engorda de camarão, com um investimento de $\mathrm{R} \$ 700.000,00$ para se obter: ponto de equilíbrio de $6,80 \%$, rentabilidade simples de $234,94 \%$, payback de 0,43 ano, TIR de 153,37\% e VPL de $\mathrm{R} \$$ 6.992.521,01 a 21\% ao ano. Todos estão sintetizados na Tabela 6:
Tabela 6. Resultados dos parâmetros econômicos para o cultivo de camarão marinho numa área de 01 hectare, no sistema de recirculação de água com o uso de BFT

\begin{tabular}{|c|c|}
\hline Parâmetros & Resultados \\
\hline Custo de Produção (R\$/ kg) & $\mathrm{R} \$ 14,29$ \\
\hline Preço Médio (R\$) & $\mathrm{R} \$ 30,00$ \\
\hline Margem de Lucro (R\$) & $\mathrm{R} \$ 15,71$ \\
\hline Ponto de equilíbrio (\%) & $6,80 \%$ \\
\hline Rentabilidade Simples (\%) & $234,94 \%$ \\
\hline Payback (ano) & 0,43 ano \\
\hline Taxa Interna de Retorno (\%) & $153,37 \%$ \\
\hline Valor Presente Líquido a 12\% a.a. (R\$) & $\mathrm{R} \$ 6.992 .521,01$ \\
\hline
\end{tabular}

Fonte: Dados da pesquisa

\section{Comparação dos dados entre os dois sistemas de cultivo estudados}

Após o processamento e sintetização dos dados levantados, obteve-se os seguintes resultados em termos de comparação entre os dois sistemas de cultivo, que foram apresentados na Tabela 7:

Tabela 7. Resultados comparativos entre os dois sistemas estudados

\begin{tabular}{|c|c|c|}
\hline Parâmetros & $\begin{array}{c}\text { Sistema semi- } \\
\text {-intensivo }\end{array}$ & $\begin{array}{c}\text { Sistema supe- } \\
\text { rintensivo }\end{array}$ \\
\hline $\begin{array}{c}\text { Custo de Produção } \\
\text { (R\$/ kg) }\end{array}$ & 17,17 & 14,29 \\
\hline Preço Médio (R\$) & 30,00 & 30,00 \\
\hline Margem de Lucro (R\$) & 12,83 & 15,71 \\
\hline Ponto de equilíbrio (\%) & 8,15 & 6,80 \\
\hline Rentabilidade Simples (\%) & 238,19 & 234,94 \\
\hline Payback (ano) & 0,42 & 0,43 \\
\hline $\begin{array}{c}\text { Taxa Interna de Retorno } \\
\text { (\%) }\end{array}$ & 153,24 & 153,37 \\
\hline $\begin{array}{c}\text { Valor Presente Líquido a } \\
12 \% \text { a.a. (R\$) }\end{array}$ & $13.847 .343,60$ & $6.992 .521,01$ \\
\hline
\end{tabular}

Fonte: Dados da Pesquisa

\section{CONCLUSÕES}

Este trabalho apresentou conceitos e metodologias de mensuração da eficiência econômica e competitividade. Além disso, descreveu as características gerais do cultivo de camarões marinhos no Brasil e dois sistemas de cultivo trabalhados: sistema de cultivo de camarão marinho tradicional (semiintensivo), sistema de cultivo de camarão marinho com recirculação de água e com uso de BFT. Também se realizou uma comparação entre os indicadores de eficiência econômica e competitividade em cada um dos sistemas de cultivo de camarões marinhos estudados. 
O sistema de cultivo semi-intensivo (tradicional) necessitou de um maior nível de investimento ( $\mathrm{R} \$ 1.350 .000,00)$ para produzir numa área de 50 hectares, com um custo de produção de $\mathrm{R} \$ 17,17$ por $\mathrm{kg}$, margem de lucro de $\mathrm{R} \$ 12,83$, ponto de equilíbrio de $8,15 \%$, rentabilidade simples de 238,19\%, payback de 0,42 ano; TIR de 153,24\% e VPL a uma taxa de $12 \%$ ao ano de $\mathrm{R} \$ \mathrm{R} \$ 13.847 .343,60$.

O sistema superintensivo necessitou de um menor nível de investimento ( $R \$ 700.000,00)$ para produzir numa área de 01 hectare, com um custo de produção de $R \$ 14,29$, margem de lucro de $\mathrm{R} \$ 15,71$, ponto de equilíbrio de $6,80 \%$, rentabilidade simples de 234,94\%, payback de 0,43 ano, TIR de $153,37 \%$ e VPL a uma taxa de $12 \%$ ao ano de $\mathrm{R} \$ \mathrm{R} \$$ 6.992.521,01. Observou-se que para terem rendimentos e parâmetros econômicos similares foi necessário que no sistema semi-intensivo (tradicional) houvesse uma área de produção de 50 hectares e investimentos da ordem de $\mathrm{R} \$$ 1.350.000,00, enquanto no sistema superintensivo, a área fosse apenas de 01 hectare e investimentos da ordem de $\mathrm{R}$ \$ 700.000,00. Embora os parâmetros econômicos tenham ficado equiparados com áreas tão distintas, a produtividade do sistema semi-intensivo foi de $6.843 \mathrm{~kg} / \mathrm{ha} / \mathrm{ano}$, enquanto no sistema superintensivo foi de $96.821 \mathrm{~kg} / \mathrm{ha} / \mathrm{ano}$.

A avaliação da eficiência produtiva pode ser orientada para o crescimento da produção: a) mantendo as quantidades de recursos constantes; b) orientando para a redução dos recursos utilizados, mantendo-se os níveis de produção; ou ainda, c) orientando para uma combinação ótima desses dois objetivos. Neste trabalho, buscou-se identificar de que forma os sistemas de cultivo em estudo poderiam obter uma maior produção, mantendo as mesmas quantidades de recursos, ou dada uma quantidade constante e limitada de recursos, maximizar a produção.

Assim, podemos concluir que o sistema de cultivo superintensivo de camarões marinhos com reuso de água e uso de BFT é, no que se refere aos parâmetros econômicos, mais eficiente e competitivo do que o cultivo no sistema semi-intensivo (tradicional).

\section{REFERÊNCIAS}

Associação Brasileira de Criadores de Camarão - ABCC. 2013. Levantamento da Infraestrutura Produtiva e dos Aspectos Tecnológicos, Econômicos, Sociais e Ambientais da Carcinicultura Marinha no Brasil em 2011. Natal, RN: ABCC. https://abccam. com.br/wp-content/uploads/2013/12/LEVANTAMENTO-DA-INFRAESTRUTURA-PRODUTIVA.pdf

Associação Brasileira de Criadores de Camarão - ABCC. 2015. Cultivo de camarão marinho: realidade mundial e brasileira, mitos e verdades sobre sua sustentabilidade ambiental e contribuição para a socioeconomia das regiões de sua intervenção. Revista da ABCC, edição especial, agosto 2015. https://abccam.com.br/wp-content/uploads/2015/08/Revista-ABCC-Edi\%C3\%A7\%C3\%A3o-Especial-Agosto-de-2015.pdf

Bastos, N.M.G. 2012. Introdução à metodologia do trabalho acadêmico. 5 ed. Fortaleza: Nacional.

Coelho, M.A.S. 2005. Análise de custo/volume/lucro e investimentos em carcinicultura de pequeno porte. Custos e @gronegócio on line 1, 62-84. http://www.custoseagronegocioonline.com.br/numero1v1/custos_na_carcinicultura.pdf

Food and Agriculture Organization of the United Nations FAO. 2020. The state of world fisheries and aquaculture 2020. Sustainability in action. Roma: FAO. https://doi.org/10.4060/ ca9229en

Gitman, L.J. 1997. Princípios de administração financeira. São Paulo: Harbra.

Hoji, M. 2001. Administração financeira, uma abordagem prática. São Paulo: Atlas.

Hopkins, J.S.; Sandifer, P.A.; Browdy, C.L. 1995. A review of water management regimes which abate the environmental impact of shrimp farming, in Browdy, C.L.; Hopkins, J.S. (Eds.). Swimming through troubled water, Baton Rouge, World Aquaculture Society.

Instituto Brasileiro de Geografia e Estatística - IBGE. 2019. IBGE Cidades. Pecuária no Brasil, 2018. https://cidades.ibge. gov.br/brasil/pesquisa/18/16459

Ito, T. 2014. O tripé de análises do IBGE. Seafood Brasil 7, 30 38. https://issuu.com/seafoodbrasil/docs/seafood brasil 7

Nascimento, I.A.; Mangabeira, F.C.; Evangelista, A.J.A. et al. 1998. Cultivo integrado de camarões e ostras: busca de uma tecnologia limpa para desenvolvimento sustentado, in Aquacultura Brasil'98, Recife, Anais... Recife: Persona, Vol. 2, pp. 503-514.

Nunes, A.J.P.; Madrid, R.M.; Andrade, T.P. 2011. Carcinicultura Marinha no Brasil: Passado, Presente e Futuro. Panorama da Aquicultura 124, 26-33. https://www.researchgate.net/publication/285763085_Carcinicultura_Marinha_no_Brasil_Passado_Presente_e_Futuro

Oliveira, R.C. 2009. O panorama da aquicultura no Brasil: a prática com foco na sustentabilidade. Revista Intertox de Toxicologia, Risco Ambiental e Sociedade 2, 71-89. https://doi. org/10.22280/revintervol2ed1.18

Otoshi, C.A.; Tang L.R.; Dagdaban, D.V. et al. 2006. Super intensive grow out of the pacific white shrimp Litopenaeus vannamei: Recent advances at the oceanic institute. In: T. Rakestraw, L. Douglas, and G. Flick (Eds.). Proceedings from the 6th International Conference on Recirculating Aquaculture. Virginia Polytechnic Institute and State University, Blacksburg, VA, pp. 1-5. 
Richardson, R.J. 1989. Pesquisa Social: Métodos e Pesquisa. 2 ed. São Paulo: Atlas.

Sampaio, L.A.; Tesser, M.B.; Wasielesky Junior, W. 2010. Avanços da maricultura na primeira década do século XXI: piscicultura e carcinocultura marinha. Revista Brasileira de Zootecnia 39, 102-111. http://dx.doi.org/10.1590/S151635982010001300012

Santos, E.; Vasan, A.H. 2014. A importância dos investimentos: uma análise por meio do Payback, VPL e TIR, in Anais dos Ciclos de Estudos em Administração e Ciclos de Estudos
Tecnológicos dos cursos de Administração e Tecnológicos da Faculdade de Jandaia do Sul - FAFIJAN, Anais... 2014.

Souza Junior, J.P. 2003. Análise da eficiência da produção de camarão marinho em cativeiro no estado do Ceará. Dissertação de Mestrado em Economia Rural, Universidade Federal do Ceará, Fortaleza, CE.

Teixeira, A.P.G.; Guerrelhas, A.C.B. 2011. Cultivo Intensivo pode ser a Solução para o Aumento da Produção da Carcinicultura? Parte II. Panorama da Aquicultura 124, 34-39. https:// panoramadaaquicultura.com.br/cultivo-intensivo-parte-2/

Recebido: 26 maio. 2020

Aprovado: 13 jul. 2020

DOI: 10.20985/1980-5160.2020.v15n2.1643

Como citar: Matias, J.F.N., Floy, H.R.F., Catter, K.M., et al. (2020). Análise comparativa da eficiência econômica e competitividade dos cultivos de camarão marinho no sistema semi-intensivo (tradicional) e superintensivo (com reuso de água e uso de bioflocos - BFT) utilizados no Brasil. Revista S\&G 15, 2, 123-130. https://revistasg. emnuvens.com.br/sg/article/view/1643 